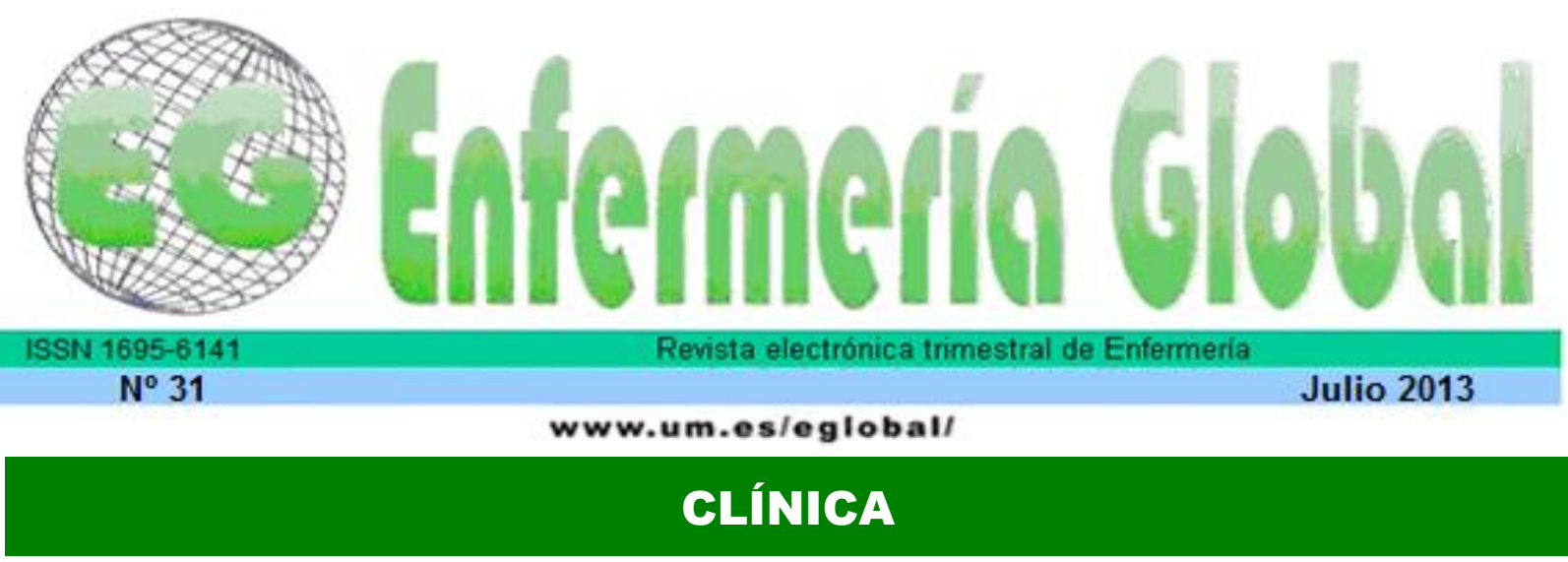

\title{
Intervenciones educativas de enfermería en pacientes ambulatorios con falla cardiaca
}

Nursing educational interventions in outpatients with heart failure

\section{${ }^{*}$ Cañón Montañez, Wilson *Oróstegui Arenas, Myriam}

*Enfermero/a, Magíster en Epidemiología. Grupo de Investigación Observatorio Epidemiológico de Enfermedades Cardiovasculares, Facultad de Salud, Universidad Industrial de Santander, Bucaramanga, Colombia. E-mail: wilcamo32@yahoo.com

Palabras clave: Evaluación de Eficacia-Efectividad de Intervenciones; Insuficiencia cardiaca; Educación; Enfermería; Ensayo Clínico controlado

Keywords: Evaluation of the Efficacy- Effectiveness of Interventions; Heart Failure; Education; Nursing; Controlled Clinical Trial..

\section{RESUMEN}

El número de pacientes con falla cardiaca cada vez es mayor. El objetivo del estudio fue determinar la eficacia de dos estrategias educativas de enfermería: (Educación Personalizada y Educación Telefónica) para aumentar el conocimiento acerca de la enfermedad en pacientes ambulatorios con falla cardiaca. Ensayo clínico aleatorizado. Análisis por intención de tratar.

Fueron aleatorizados 116 pacientes. En el grupo que recibió intervención educativa personalizada, el delta del puntaje de conocimiento aumentó en la evaluación final en 1.04 (IC 95\%: 0.94; 1.14); y en el seguimiento en 0.73 (IC 95\%: 0.63; 0.83). Asimismo, para el grupo asignado a intervención educativa telefónica, el delta del puntaje aumentó en la evaluación final en 1.00 (IC 95\%: 0.92; 1.07); y en el seguimiento, en 0.73 (IC 95\%: 0.64; 0.81).

Ambas intervenciones tuvieron un efecto benéfico. La educación de enfermería en este tipo de pacientes, independientemente de la estrategia (personalizada o telefónica) es útil y por lo tanto los profesionales de enfermería deberían ser incluidos en los equipos multidisciplinarios de atención. (Registro Latinoamericano de Ensayos Clínicos en Curso: COL112).

\section{ABSTRACT}

The number of patients with heart failure is increasing. The purpose of this study was to determine the efficacy of two nursing educational strategies (Personalized Education and Phone Education) to raise the knowledge about the disease in outpatients with heart failure. Controlled clinical trial. Analysis was by intention to treat. 
116 patients were randomized. In the group who received personalized educational intervention, the delta score of knowledge increased in the final evaluation at $1.04(95 \% \mathrm{Cl}: 0.94,1.14)$, and in the follow-up at $0.73(95 \% \mathrm{Cl}: 0.63,0.83)$. Also, for the group assigned to educational by phone, the delta score of knowledge increased in the final evaluation at 1.00 (95\% Cl: $0.92,1.07)$, and in the follow-up at 0.73 (95\% Cl: 0.64, 0.81).

Both interventions had a beneficial effect. Nursing education in these patients, regardless of the strategy (personalized or by phone) is useful and therefore nurses should be included in the multidisciplinary teams of care. (Latin American Clinical Trials Register: COL112).

\section{INTRODUCCIÓN}

La falla cardiaca (FC) es considerada actualmente como uno de los problemas de salud pública más frecuente y que los profesionales sanitarios deben abordar. Se ha investigado en extenso la fisiopatología, etiología, diagnóstico y tratamiento; sin embargo, en las últimas dos décadas la prevalencia e incidencia de la enfermedad ha aumentado de manera relevante y se ha indicado que la mortalidad a corto y largo plazo asociada a esta entidad en algunos casos supera la tasa observada en algunos cánceres ${ }^{(1)}$. Además, el descompensamiento de la enfermedad se ha asociado con hospitalizaciones frecuentes, mala calidad de vida, baja adherencia al tratamiento y un exceso en los costos para su manejo ${ }^{(2)}$.

En el manejo de los pacientes con FC es importante no sólo medicarlos sino brindarles una atención integral con colaboración multidisciplinaria. Al respecto, hoy en día, en la mayoría de países industrializados se han habilitado unidades especializadas, con equipos terapéuticos multidisciplinarios para el manejo y abordaje de los pacientes con FC, en los cuales enfermería juega un rol importante con intervenciones de seguimiento, cuidado y educación de los pacientes. Estas unidades se han denominado "Clínicas de FC" (3). En Bucaramanga - Colombia, hay incertidumbre porque existen muy pocas de estas unidades y no se conocen estudios que evalúen la eficacia de estas intervenciones.

Existe evidencia de que los factores asociados a un bajo nivel de conocimiento y a una pobre adherencia al tratamiento son: desconocimiento del proceso de la enfermedad, incumplimiento del régimen terapéutico, falta de apoyo social y seguimiento inadecuado ${ }^{(4)}$. De manera que la educación al paciente es un componente clave en el manejo de la FC ${ }^{(5)}$. Cabe señalar que en la educación al paciente con FC, los profesionales de enfermería deben tener la habilidad para evaluar las necesidades individuales y comenzar el proceso de educación basado en el nivel de percepción previo del paciente con relación a la enfermedad. Adicionalmente, el compromiso del enfermo en el proceso educativo es un factor primordial para obtener los resultados esperados ${ }^{(6)}$.

Ahondando al respecto, es importante también, evaluar los efectos de la educación. Los desenlaces que son relevantes medir, dependen de lo que se espera alcanzar con la educación ofrecida. El conocimiento acerca de la enfermedad es difícil de medir y hay pocos instrumentos estandarizados desarrollados para medir el conocimiento en los pacientes con FC ${ }^{(7)}$. Dentro de este contexto, es importante mencionar que la enfermería es una profesión privilegiada pues cuenta con un lenguaje estandarizado propio con taxonomías para diagnósticos ${ }^{(8)}$, intervenciones ${ }^{(9)}$ y resultados ${ }^{(10)}$. En relación a la $\mathrm{FC}$, la enfermería tiene en la taxonomía de resultados una etiqueta específica para evaluar el conocimiento acerca de la enfermedad, denominada: Conocimiento: control de la enfermedad cardiaca ${ }^{(10)}$. 
Estudios previos han establecido qué estrategias educativas multifactoriales que incluyen instrucción personalizada y seguimiento telefónico frecuente han demostrado ser eficaces para mejorar el conocimiento acerca de la enfermedad y disminuir la tasa de ingresos hospitalarios ${ }^{(11)}$. Sin embargo, hay incertidumbre del efecto real de estas intervenciones por el carácter multifactorial de la educación ${ }^{(12)}$.

El objetivo de este estudio fue determinar la eficacia de una intervención educativa personalizada de enfermería comparada con una intervención educativa telefónica de enfermería, para aumentar el conocimiento acerca de la enfermedad en pacientes ambulatorios con diagnóstico de FC.

\section{MÉTODOS}

\section{Diseño, Población de estudio y Tamaño de muestra}

Estudio experimental tipo ensayo clínico controlado aleatorizado de tipo paralelo. El diseño y protocolo del estudio fue registrado en el Registro Latinoamericano de Ensayos Clínicos en Curso, código: COL112. Para el reporte de los resultados del presente estudio se tuvo en cuenta la extensión de la Declaración CONSORT para ensayos clínicos aleatorizados de intervenciones no farmacológicas ${ }^{(13)}$.

La población de estudio estuvo conformada por pacientes con diagnóstico de FC asistentes a consulta ambulatoria de cardiología clínica de un hospital universitario. El diagnóstico de FC fue realizado por el médico tratante de los participantes de acuerdo a los protocolos y guías de manejo de la institución. Los criterios de inclusión para participar en el estudio fueron: personas mayores de 18 años residentes en Bucaramanga o su área metropolitana con diagnóstico de FC clase funcional New York Heart Association (NYHA) I, II, III ó IV; puntuación inicial menor o igual a 3.0 en la etiqueta resultado de enfermería: Conocimiento: control de la enfermedad cardiaca (1830) de la Clasificación de Resultados de Enfermería (CRE) ${ }^{(10)}$. Fueron excluidos pacientes con trastornos psiquiátricos o conductuales que dificultaran la comprensión de las intervenciones; pacientes con otras comorbilidades que limitaran recibir la intervención (ej: hipoacusia, cofosis, enfermedad de Alzheimer, deterioro cognitivo o déficit de memoria) y pacientes que no disponían de telefonía fija o móvil.

De acuerdo a la revisión de parámetros estadísticos para el cálculo del tamaño muestral y de los resultados de estudios similares publicados previamente ${ }^{(14)}$, fue calculado un tamaño de muestra de $n=58$ sujetos por grupo (total $n=116$ ), de acuerdo a los siguientes parámetros: tamaño del efecto: un delta o diferencia esperada de 0.5 en el puntaje de resultado de la evaluación del conocimiento entre ambos grupos, una potencia del 90\%, un error tipo alfa del 5\%, una desviación estándar de las puntuaciones de resultado de 1.0, un promedio de correlaciones entre primera y segunda evaluación de 0.3, una razón intervenido/control de $1: 1$ y un ajuste por pérdidas del $20 \%$. Se utilizó para este fin el programa STATA® $11.0^{(15)}$.

\section{Intervenciones educativas (Grupo intervención y Grupo control)}

El grupo de intervención además del manejo usual brindado en el hospital, recibió una intervención educativa personalizada ofrecida por un profesional de enfermería. Esta intervención no corresponde a ninguna etiqueta de la Clasificación de Intervenciones de Enfermería (CIE) ${ }^{(9)}$ y fue elaborada y estandarizada por los investigadores de acuerdo al análisis y revisión de la evidencia sobre lo que debe conocer un paciente 
con diagnóstico de falla cardiaca acerca de su enfermedad ${ }^{(6)}$. La intervención tuvo una duración de 30 minutos y todos los pacientes recibieron tres sesiones de intervención. La intervención educativa personalizada fue definida como la enseñanza cara a cara sin contacto telefónico ofrecida por enfermería al paciente acerca de qué es la falla cardiaca, cómo lograr la capacidad de autoayuda, detección de señales y síntomas de descompensación de la enfermedad y búsqueda precoz de los servicios de salud.

El grupo control además del manejo usual brindado en el hospital, recibió la misma intervención educativa del grupo de intervención pero mediante vía telefónica.

\section{Desenlace principal y otros desenlaces secundarios}

El desenlace principal fue la puntuación promedio final de cada paciente en la etiqueta resultado CRE ${ }^{(10)}$ Conocimiento: control de la enfermedad cardiaca, al mes (CRE Final) y a los dos meses posteriores al inicio de las intervenciones (CRE Seguimiento). Otros desenlaces secundarios evaluados fueron: mortalidad total, mortalidad por FC, hospitalización por descompensación de la FC, frecuencia de hospitalización por FC y estancia hospitalaria por FC.

\section{Aleatorización, Enmascaramiento y Seguimiento}

Se realizó asignación aleatoria simple en grupos de igual tamaño. La generación de la secuencia fue realizada por el Investigador Principal, quien no participó en las intervenciones ni en la evaluación del desenlace. Se utilizó para este fin el programa EPIDAT $3.1^{(16)}$. Los pacientes fueron aleatorizados $1: 1$ a recibir educación personalizada o educación telefónica.

Se efectuó el siguiente enmascaramiento: la enfermera que realizó las intervenciones fue enmascarada al desenlace principal del estudio; los enfermeros que evaluaron el desenlace principal y el investigador que realizó el análisis de los datos fueron enmascarados al grupo (intervención o control) que pertenecían los pacientes.

Todos los pacientes fueron seguidos al mes y a los dos meses después de haber iniciado las intervenciones.

\section{Instrumento}

Para evaluar el conocimiento acerca de la FC en los participantes, se utilizó la etiqueta resultado CRE ${ }^{(10)}$ Conocimiento: control de la enfermedad cardiaca, que corresponde al código 1830 de la CRE, consta de 27 indicadores con escala tipo Likert con una puntuación de 1 a 5 (1=ninguno, 2=escaso, 3=moderado, 4=sustancial y $5=$ =xtenso), y está definida como el grado de la comprensión transmitida sobre la enfermedad cardiaca y la prevención de complicaciones. Previamente y mediante el consenso de dos profesionales de enfermería expertos en el área cardiovascular, se realizó la operacionalizacion de seis indicadores de la etiqueta resultado CRE ${ }^{(10)}$ Conocimiento: control de la enfermedad cardiaca.

\section{Procedimientos}

Previo a la realización de las intervenciones objeto de estudio fue realizada la firma voluntaria del consentimiento informado. Dos evaluadores enmascarados a las 
estrategias de intervención y entrenados previamente durante dos semanas en el manejo, utilización y aplicación de la etiqueta resultado de enfermería CRE, hicieron la valoración acerca del conocimiento de la enfermedad de los participantes al inicio (CRE Inicial previo a la intervención educativa), al mes (CRE Final) y a los dos meses después de haber iniciado las intervenciones (CRE Seguimiento). La enfermera que realizó las intervenciones no tuvo influencia en la evaluación del desenlace principal. Adicionalmente los investigadores, una vez finalizado el estudio entregaron material educativo impreso relacionado con la enfermedad, en agradecimiento a los participantes.

\section{Procesamiento y Análisis de los Datos}

Los datos generados se registraron en formatos previamente diseñados y estandarizados para tal fin. Todos los registros fueron sometidos a doble digitación de la información con posterior contrastación de posibles inconsistencias y errores de digitación en el programa estadístico EPIDATA $3.1^{(16)}$.

Se siguió el principio de análisis por intención de tratamiento. Todas las pruebas de hipótesis estadísticas se hicieron a un nivel alfa de $5 \%$. Los ajustes y procedimientos estadísticos fueron documentados en Stata ${ }^{\circledR} 11.0^{(15)}$. Se realizó un análisis descriptivo para comparar las características basales entre el grupo intervención y el grupo control. Las variables fueron comparadas entre los grupos por medio de la pruebas $t$ de Student y exacta de Fisher ${ }^{(17)}$. Las variables categóricas se presentaron por medio de frecuencias absolutas y relativas; las variables continuas con distribución normal se presentaron con media y desviación estándar, aquellas que no presentaron una distribución normal se describieron con la mediana y el rango intercuartil. Para evaluar el efecto de las intervenciones sobre el promedio de las puntuaciones finales de los grupos se realizó un análisis de covarianza (ANCOVA) ${ }^{(18)}$.

\section{Consideraciones Éticas}

El protocolo y diseño del estudio fue aprobado en sus aspectos técnicos-científicos por el Comité de Ética para la Investigación Científica de la Facultad de Salud de la Universidad Industrial de Santander bajo el código: EP10019. Asimismo la presente investigación fue avalada por la Oficina de Calidad del hospital universitario donde se reclutaron los participantes. La participación de los individuos en la investigación fue de manera voluntaria. Para ingresar al estudio se solicitó el consentimiento informado escrito del paciente. Todos los procedimientos fueron de acuerdo con los principios establecidos en la Declaración de Helsinki de la Asociación Médica Mundial ${ }^{(19)}$, del Reporte Belmont ${ }^{(20)}$, de las Pautas Éticas Internacionales para la Investigación Biomédica en Seres Humanos ${ }^{(21)}$ preparadas por el Consejo de Organizaciones Internacionales de Ciencias Médicas (CIOMS) en colaboración con la Organización Mundial de la Salud (OMS), las Guías de las Buenas Prácticas Clínicas de la Conferencia Internacional de Armonización ${ }^{(22)}$ y las Normas Científicas, Técnicas y Administrativas para la Investigación en Salud de la Resolución 008430 del Ministerio de Salud de la República de Colombia ${ }^{(23)}$.

\section{RESULTADOS}

Entre septiembre de 2010 y febrero de 2011 se evaluaron 242 pacientes ambulatorios con diagnóstico de FC en un hospital universitario en Bucaramanga -Colombia. Del total de los pacientes evaluados 157 (64.8\%) cumplieron criterios de inclusión. Se 
excluyeron a 41 (26.1\%) de los pacientes elegibles por presentar trastornos psiquiátricos conductuales, hipoacusia, déficit de memoria, no disponer de telefonía fija o móvil y por no aceptar participar para ingresar al estudio. En total fueron aleatorizados 116 pacientes $(73.8 \%), 58$ a recibir intervención educativa personalizada y 58 a recibir intervención educativa telefónica. Todos los pacientes asignados a intervención educativa telefónica recibieron las tres sesiones educativas planeadas. Por el contrario, del total de participantes que recibieron intervención educativa personalizada, 56 pacientes $(96.5 \%)$ recibieron tres sesiones educativas, y 02 pacientes (3.5\%) recibieron sólo una sesión. (Figura 1). 
Figura No. 1 Diagrama de flujo de los participantes

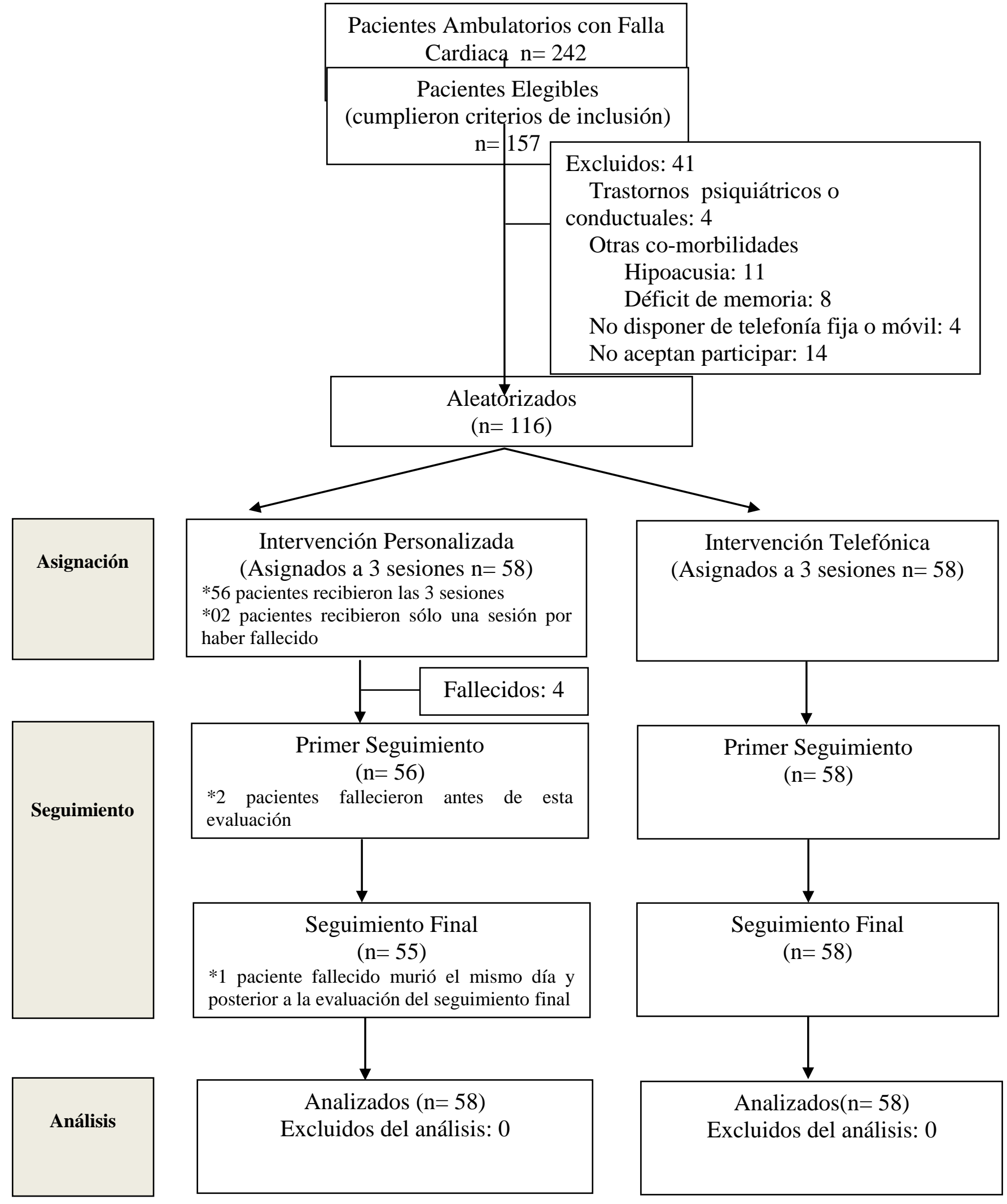




\section{Características Basales de los Participantes}

Las características sociodemográficas y clínicas de los participantes en los dos grupos de intervención son resumidas en la Tabla No. 01. Las variables continuas tuvieron una distribución no normal y son presentadas con la mediana y rango intercuartil. La normalidad de las variables continuas fue evaluada mediante la prueba de Shapiro Wilk ${ }^{(24)}$. Las variables categóricas se presentan por medio de frecuencias absolutas y relativas. Todas las variables basales se distribuyeron de forma balanceada y homogénea y no se presentaron diferencias estadísticamente significativas entre los grupos de intervención.

Tabla No. 1 Características basales (variables sociodemográficas y clínicas) de los pacientes

\begin{tabular}{|c|c|c|}
\hline Variable & $\begin{array}{l}\text { Intervención Personalizada } \\
\qquad \begin{array}{c}(\mathbf{n}=\mathbf{5 8}) \\
\mathbf{n}(\%)\end{array} \\
\end{array}$ & $\begin{array}{c}\begin{array}{c}\text { Intervención Telefónica } \\
(\mathbf{n}=\mathbf{5 8}) \\
\mathbf{n}(\%) \\
\end{array} \\
\end{array}$ \\
\hline Edad (años cumplidos)* & $66(20)$ & $70(21)$ \\
\hline \multicolumn{3}{|l|}{ Sexo } \\
\hline Hombres & $27(53.45)$ & $27(53.45)$ \\
\hline Mujeres & $31(46.55)$ & $31(46.55)$ \\
\hline \multicolumn{3}{|l|}{ Estado Civil } \\
\hline Soltero & $6(10.34)$ & $4(6.90)$ \\
\hline Casado & $26(44.83)$ & $24(41.38)$ \\
\hline Unión Libre & $6(10.34)$ & $7(12.07)$ \\
\hline Separado/Divorciado & $0(0.00)$ & $3(5.17)$ \\
\hline Viudo & $20(34.48)$ & $20(34.48)$ \\
\hline \multicolumn{3}{|l|}{ Ocupación } \\
\hline Estudiante & $1(1.72)$ & $0(0.00)$ \\
\hline Empleado & $0(0.00)$ & $1(1.72)$ \\
\hline Independiente & $19(32.76)$ & $10(17.24)$ \\
\hline Desempleado/Cesante & $37(63.39)$ & $47(81.03)$ \\
\hline Otro & $1(1.72)$ & $0(0.00)$ \\
\hline \multicolumn{3}{|l|}{ Estrato } \\
\hline Uno & $38(65.52)$ & $30(51.72)$ \\
\hline Dos & $17(29.31)$ & $26(44.83)$ \\
\hline Tres & $3(5.17)$ & $2(3.45)$ \\
\hline \multicolumn{3}{|l|}{ Residencia } \\
\hline Urbana & $51(87.93)$ & $49(84.48)$ \\
\hline Rural & $7(12.07)$ & $9(15.52)$ \\
\hline \multicolumn{3}{|l|}{ Seguridad Social } \\
\hline Régimen Subsidiado & $58(100.00)$ & $58(100.00)$ \\
\hline Escolaridad (años cursados y aprobados)* & $2(4)$ & $2(4)$ \\
\hline \multicolumn{3}{|l|}{ Soporte de Cuidador } \\
\hline Pareja & $23(44.23)$ & $20(42.55)$ \\
\hline Padres & $1(1.92)$ & $0(0.00)$ \\
\hline Hermanos & $2(3.85)$ & $2(4.26)$ \\
\hline Otro Familiar & $29(55.77)$ & $27(57.45)$ \\
\hline \multicolumn{3}{|l|}{ Etiología de la Falla Cardiaca } \\
\hline Isquémica & $12(20.69)$ & $11(18.97)$ \\
\hline Hipertensiva & $12(20.69)$ & $10(17.24)$ \\
\hline Valvular & $11(18.97)$ & $22(37.93)$ \\
\hline Chagásica & $12(20.69)$ & $6(10.34)$ \\
\hline Congénita & $0(0.00)$ & $2(3.45)$ \\
\hline Desconocida & $9(15.52)$ & $4(6.90)$ \\
\hline Otra & $2(3.45)$ & $3(5.17)$ \\
\hline Tiempo de Diagnóstico de la Falla Cardiaca (meses)* & $48(60)$ & $45(67)$ \\
\hline \multicolumn{3}{|l|}{ Clase Funcional New York Heart Association NYHA } \\
\hline I & $1(1.72)$ & $0(0.00)$ \\
\hline II & $16(27.59)$ & $18(31.03)$ \\
\hline III & $40(68.97)$ & $38(65.52)$ \\
\hline IV & $1(1.72)$ & $2(3.45)$ \\
\hline
\end{tabular}




\begin{tabular}{lcc}
\hline Estadio de la Falla Cardiaca & $33(56.90)$ \\
$\mathrm{B}$ & $31(53.45)$ & $25(43.10)$ \\
$\mathrm{C}$ & $27(46.55)$ & $35(25)$ \\
\hline Fracción de Eyección del Ventrículo Izquierdo FEVI & $35(20)$ & $8(16.33)$ \\
$(\%)^{*}$ & & $39(79.59)$ \\
\hline Comorbilidad & $5(11.90)$ & $2(4.08)$ \\
$\quad$ Diabetes & $32(76.19)$ & $7(14.29)$ \\
$\quad$ Hipertensión & $2(4.76)$ &
\end{tabular}

\section{Efecto de la Educación entre los Grupos de Intervención}

En el análisis bivariado se observaron diferencias estadísticamente significativas de los puntajes promedio de la etiqueta resultado de enfermería: Conocimiento: control de la enfermedad cardiaca (1830), entre el CRE Inicial, CRE Final, CRE Seguimiento dentro de los grupos de intervención educativa personalizada e intervención educativa telefónica. Estas diferencias fueron determinadas mediante pruebas $t$ de Student pareadas. En la Tabla No. 2 se puede observar cómo se logra un aumento importante del puntaje promedio del conocimiento en ambos grupos de intervención. Asimismo, las Tablas No. 3 y 4 nos muestran cómo se mantiene el efecto de las intervenciones en el seguimiento aún después que las intervenciones habían terminado.

Tabla No. 2 Diferencia entre el puntaje promedio inicial y final de cada grupo de la etiqueta resultado de enfermería: Conocimiento: control de la enfermedad cardiaca (1830)

\begin{tabular}{|c|c|c|c|c|}
\hline Grupo & $\begin{array}{c}\text { Puntaje } \\
\text { Promedio } \\
\text { CRE Inicial }\end{array}$ & $\begin{array}{l}\text { Puntaje } \\
\text { Promedio } \\
\text { CRE Final }\end{array}$ & $\begin{array}{l}\text { Diferencia del } \\
\text { puntaje promedio } \\
\text { CRE Final y CRE } \\
\text { Inicial }\end{array}$ & $\begin{array}{c}\text { Valor de } \\
\text { p }^{*}\end{array}$ \\
\hline $\begin{array}{l}\text { Intervención } \\
\text { Educativa } \\
\text { Personalizada }\end{array}$ & $1.81(1.73 ; 1.88)$ & $\begin{array}{c}2.85(2.74 ; \\
2.96)\end{array}$ & $1.04(0.94 ; 1.14)$ & $<0.0001$ \\
\hline $\begin{array}{l}\text { Intervención } \\
\text { Educativa } \\
\text { Telefónica }\end{array}$ & $1.81(1.74 ; 1.88)$ & $\begin{array}{l}2.81(2.71 ; \\
2.91)\end{array}$ & $1.00(0.92 ; 1.07)$ & $<0.0001$ \\
\hline \multicolumn{5}{|c|}{$\begin{array}{l}\text { Tabla No. } 3 \text { Diferencia entre el puntaje promedio inicial y de seguimiento ( } 2 \text { meses post } \\
\text { inicio de intervención) de cada grupo de la etiqueta resultado de enfermería: } \\
\text { Conocimiento: control de la enfermedad cardiaca }(1830)\end{array}$} \\
\hline Grupo & $\begin{array}{l}\text { Puntaje } \\
\text { Promedio } \\
\text { CRE Inicial }\end{array}$ & $\begin{array}{l}\text { Puntaje } \\
\text { Promedio } \\
\text { CRE } \\
\text { Seguimiento }\end{array}$ & $\begin{array}{c}\text { Diferencia del } \\
\text { puntaje promedio } \\
\text { CRE Seguimiento y } \\
\text { CRE Inicial }\end{array}$ & $\begin{array}{c}\text { Valor de } \\
\text { p }^{*}\end{array}$ \\
\hline $\begin{array}{c}\text { Intervención } \\
\text { Educativa } \\
\text { Personalizada }\end{array}$ & $1.82(1.74 ; 1.89)$ & $\begin{array}{c}2.55(2.45 ; \\
2.66)\end{array}$ & $0.73(0.63 ; 0.83)$ & $<0.0001$ \\
\hline
\end{tabular}


Intervención

Educativa

$1.81(1.74 ; 1.88)$

$2.54(2.42$

$0.73(0.64 ; 0.81)$

$<0.0001$

Telefónica

$2.65)$

Valores expresados como media (intervalo de confianza del 95\%). CRE: Clasificación de resultados de enfermería.

*Prueba t de Student pareada.

Tabla No. 4 Diferencia entre el puntaje promedio final y de seguimiento ( 2 meses post inicio de intervención) de cada grupo de la etiqueta resultado de enfermería: Conocimiento: control de la enfermedad cardiaca (1830)

\begin{tabular}{ccccc}
\hline Grupo & $\begin{array}{c}\text { Puntaje } \\
\text { Promedio } \\
\text { CRE Final }\end{array}$ & $\begin{array}{c}\text { Puntaje } \\
\text { Promedio } \\
\text { CRE } \\
\text { Seguimiento }\end{array}$ & $\begin{array}{c}\text { Diferencia del } \\
\text { puntaje promedio } \\
\text { CRE Seguimiento y } \\
\text { CRE Final }\end{array}$ & $\begin{array}{c}\text { Valor de } \\
\mathbf{p}^{*}\end{array}$ \\
\hline $\begin{array}{c}\text { Intervención } \\
\text { Educativa }\end{array}$ & $2.85(2.74 ; 2.96)$ & $\begin{array}{c}2.55(2.45 ; \\
2.66)\end{array}$ & $\mathbf{0 . 3 0}(\mathbf{0 . 2 4} ; \mathbf{0 . 3 4})$ & $<0.0001$ \\
$\begin{array}{c}\text { Personalizada } \\
\text { Intervención }\end{array}$ & & & & \\
Educativa & $2.81(2.71 ; 2.91)$ & $2.54(2.42 ;$ & $\mathbf{0 . 2 7}(\mathbf{0 . 2 2} ; \mathbf{0 . 3 2})$ & $<0.0001$ \\
Telefónica & & $2.65)$ & & \\
\hline Valores expresados como media (intervalo de confianza del 95\%). CRE: Clasificación de resultados de enfermería.
\end{tabular}

*Prueba t de Student pareada.

\section{Comparación y efecto de las intervenciones}

Previamente se documentó que los pacientes que recibieron tanto educación de enfermería personalizada como los que recibieron educación de enfermería telefónica acerca de la FC, aumentaron el puntaje en la evaluación del conocimiento de la etiqueta resultado de enfermería en el CRE Final y el efecto se mantuvo aunque disminuido en el CRE Seguimiento, respecto al puntaje del CRE Inicial. Sin embargo, al comparar y evaluar el efecto entre los dos grupos, el análisis de covarianza mostró que no hubo diferencia entre las dos intervenciones de estudio y por lo tanto no se pudo demostrar que la intervención educativa de enfermería personalizada es más eficaz que la intervención educativa de enfermería telefónica (Tablas No. 5, 6 y 7).

Tabla No. 5 Análisis del efecto de las intervenciones entre los grupos en la etiqueta CRE final ajustado por el CRE inicial (Análisis de Covarianza ANCOVA)

$\begin{array}{llll}\text { Etiqueta CRE Final } & \text { Delta } & \text { Valor de p } & \text { IC 95\% }\end{array}$

$\begin{array}{llllll}\text { Conocimiento: } & \text { control de la } & 0.038 & 0.536 & -0.084 ; 0.160\end{array}$

enfermedad cardiaca (1830)

CRE: Clasificación de resultados de enfermería. IC 95\%: Intervalo de confianza del $95 \%$. 
Tabla No. 6 Análisis del efecto de las intervenciones entre los grupos en la etiqueta CRE seguimiento ajustado por el CRE inicial (Análisis de Covarianza ANCOVA)

$\begin{array}{llll}\text { Etiqueta CRE Seguimiento } & \text { Delta } & \text { Valor de p } & \text { IC 95\% }\end{array}$
Conocimiento: control de la
$-0.014$
0.834
$-0.115 ; 0.143$

enfermedad cardiaca (1830)

CRE: Clasificación de resultados de enfermería. IC 95\%: Intervalo de confianza del 95\%.

Tabla No. 7 Análisis del efecto de las intervenciones entre los grupos en la etiqueta CRE seguimiento ajustado por el CRE final (Análisis de Covarianza ANCOVA)
Etiqueta CRE Seguimiento
Delta
Valor de p
IC 95\%

$\begin{array}{llllll}\text { Conocimiento: } & \text { control de la } & -0.015 & 0.676 & -0.085 ; 0.055\end{array}$

enfermedad cardiaca (1830)

CRE: Clasificación de resultados de enfermería. IC 95\%: Intervalo de confianza del 95\%.

\section{Otros desenlaces secundarios de interés}

Otros desenlaces que se quiso evaluar en el seguimiento final fue lo relacionado a muerte debido a la FC, hospitalizaciones y estancia hospitalaria por descompensación de la enfermedad. En la Tabla No. 8, se puede observar que todas las defunciones debido a la FC ocurrieron en el grupo de intervención educativa de enfermería personalizada. Asimismo, se encontró que hubo más pacientes que se hospitalizaron por descompensación de la enfermedad en el grupo de intervención personalizada. En contraste, el promedio de días de estancia hospitalaria por descompensación de la FC fue mayor en el grupo de intervención telefónica. Ninguno de estos hallazgos entre los grupos de intervención fue estadísticamente significativo.

Tabla No. 8 Otros desenlaces secundarios en el seguimiento final ( 2 meses post inicio de intervenciones)

\begin{tabular}{|c|c|c|c|}
\hline Variable & $\begin{array}{c}\text { Intervención Personalizada } \\
(\mathbf{n}=\mathbf{5 8}) \\
\mathbf{n}(\%) \\
\end{array}$ & $\begin{array}{c}\begin{array}{c}\text { Intervención Telefónica } \\
(\mathbf{n}=58) \\
\mathbf{n}(\%)\end{array} \\
\end{array}$ & $\begin{array}{c}\text { Valor de } \\
\text { p }\end{array}$ \\
\hline Fallecimientos (por falla cardiaca) & $4(6.90)$ & $0(0.00)$ & $0.119 \dagger$ \\
\hline $\begin{array}{l}\text { Hospitalización por Descompensación de la Falla } \\
\text { Cardiaca }\end{array}$ & $11(18.97)$ & $5(8.62)$ & $0.177 \dagger$ \\
\hline $\begin{array}{lccc}\text { Días Totales de } & \text { Hospitalización } & \text { por } \\
\text { Descompensación de la Falla Cardiaca } & \\
\end{array}$ & $69(55.65)$ & $55(44.35)$ & $0.507 \dagger$ \\
\hline $\begin{array}{lccc}\text { Promedio } & \text { de } & \text { Hospitalizaciones } & \text { por } \\
\text { Descompensación de la Falla Cardiaca* } & \\
\end{array}$ & $1.18(0.40)$ & $1.20(0.44)$ & $0.936 \ddagger$ \\
\hline $\begin{array}{l}\text { Promedio de Días de Estancia Hospitalaria por } \\
\text { Descompensación de la Falla Cardiaca* }\end{array}$ & $6.27(5.93)$ & $11(11)$ & $0.275 \$$ \\
\hline
\end{tabular}




\section{DISCUSIÓN}

Este es el primer ensayo clínico aleatorizado de Colombia que evalúa la eficacia y el efecto de intervenciones educativas de enfermería para aumentar el conocimiento acerca de la enfermedad en pacientes ambulatorios con FC. Adicionalmente, es el primer estudio que evalúa el conocimiento acerca de la FC usando una etiqueta resultado de enfermería 'específica' para personas con enfermedades cardiovasculares, denominada: Conocimiento: control de la enfermedad cardiaca (1830), de la Clasificación de Resultados de Enfermería CRE. En la revisión de antecedentes investigativos para evaluar el conocimiento en población con $\mathrm{FC}$ y que hayan usado etiquetas resultado CRE, sólo se encontró el estudio de Yera-Casas et al (11), el cual evaluó el conocimiento en los pacientes mediante las etiquetas resultados CRE: Conocimiento: proceso de la enfermedad (1803), Conocimiento: dieta (1802), Conocimiento: medicación (1808) y Conocimiento: actividad prescrita (1811).

Los resultados del presente estudio no logran demostrar que una intervención educativa de enfermería personalizada es más eficaz que una intervención educativa de enfermería telefónica para aumentar el conocimiento acerca de la enfermedad en pacientes ambulatorios con FC. A pesar de ello, este estudio documenta que ambas intervenciones por separado producen un efecto benéfico relevante, evidenciado en el aumento del delta del puntaje promedio de conocimiento alcanzado post-intervención y que este efecto se mantiene aunque disminuido hasta dos meses después de haber iniciado las intervenciones. Esto se relaciona con los resultados del estudio de YeraCasas et al ${ }^{(11)}$, quienes lograron aumentar el delta del conocimiento post-intervención en los pacientes con FC entre 1.6 y 2.2, en las etiquetas de resultado CRE, que los investigadores evaluaron. En este sentido, estos hallazgos también se relacionan con otros estudios que aunque no usaron etiquetas resultado CRE, también encontraron diferencias estadísticamente significativas en el aumento del conocimiento de los pacientes acerca de la FC entre los grupos de comparación ${ }^{(25)}$.

En relación a la importancia de evaluar o medir el conocimiento de los pacientes con FC, los resultados del presente estudio aportan a la evidencia y se relacionan con las contribuciones de Strömberg ${ }^{(26)}$, quien afirma que se requiere más investigación que use métodos de evaluación de los niveles de conocimiento y las habilidades cognitivas en pacientes con FC. De igual manera, es importante mencionar las conclusiones de Agård et al ${ }^{(27)}$, quienes argumentan que los pacientes con $\mathrm{FC}$ están debidamente informados cuando han alcanzado el nivel de conocimiento que les permita manejar su enfermedad y mientras estén satisfechos con la información proporcionada. Para dar una información adecuada, los proveedores de salud deben determinar el nivel previo de conocimientos y explorar por qué los pacientes que tienen una comprensión limitada no se asimilan o solicitan mayor información.

En este ensayo clínico controlado, la asignación aleatoria simple logró que las características basales y otras variables clínicas estudiadas potencialmente confusoras, quedaran distribuidas de forma balanceada y homogénea entre los grupos de intervención. Aunque no hubo diferencias estadísticamente significativas en la distribución de la variables entre los grupos de comparación, debido a la aleatorización; se realizaron pruebas t de Student pareadas entre los puntajes promedio de la etiqueta resultado de enfermería: Conocimiento: control de la enfermedad cardiaca (1830), en el CRE Inicial, CRE Final, y CRE Seguimiento. Los resultados mostraron diferencias estadísticamente significativas que deberían ser 
tenidas en cuenta si se quisieran implementar programas multidisciplinarios de atención con soporte educativo para pacientes con FC.

Asimismo, es de destacar los resultados de van der Wal et al ${ }^{(28)}$, quienes realizaron un estudio con el objetivo de evaluar todas las dimensiones de la adherencia al tratamiento y sus factores relacionados en una población de pacientes con FC. Los principales hallazgos mostraron que la adherencia al tratamiento fue asociada con el conocimiento de los pacientes acerca de la enfermedad (OR: 5.67; IC95\% 2.87 11.19).

Otros desenlaces que se quiso evaluar en el seguimiento final (2 meses post-inicio de intervenciones) y aunque no fueron objetivos principales, fue lo relacionado a muerte debido a la FC, hospitalizaciones y estancia hospitalaria por descompensación de la enfermedad. Los resultados muestran que todos los pacientes fallecidos fueron del grupo que recibió educación personalizada. Igualmente, hubo más pacientes hospitalizados por descompensación de la enfermedad en este grupo. En contraste, el promedio de días de estancia hospitalaria fue mayor para los pacientes que recibieron educación telefónica. Es de aclarar que ninguna de estas diferencias fue estadísticamente significativa y que para el cálculo del tamaño muestral no se tuvo en cuenta estos desenlaces. Al respecto conviene decir y según lo reportado por otros estudios, que se requiere más tiempo de seguimiento para encontrar diferencias en mortalidad y reingresos hospitalarios por FC. Ejemplo de ello son los reportes de algunos estudios en este tipo de población ${ }^{(29)}$, que no han encontrado diferencias estadísticamente significativas en mortalidad y reingresos hospitalarios por descompensación de la enfermedad, adherencia al tratamiento y calidad de vida, en periodos de seguimiento que oscilan entre 2 a 12 meses.

Una posible limitación de este estudio es no haber tenido un grupo control que no hubiera recibido ninguna intervención y con el cual se haya podido ajustar en el análisis de covarianza para determinar la potencial eficacia de las intervenciones objeto de estudio. Sin embargo, la revisión de la literatura y los antecedentes investigativos evidenciaron que este tipo de intervenciones por separado comparadas con no recibir intervención o recibir el cuidado habitual en el hospital ya habían demostrado ser eficaces, y por lo tanto posiblemente no era ético para el presente estudio dejar a un tercer grupo sin recibir intervención.

\section{CONCLUSIONES}

En este ensayo clínico aleatorizado no se demostró que una intervención educativa de enfermería personalizada es más eficaz que una intervención educativa de enfermería telefónica para aumentar el conocimiento acerca de la enfermedad en pacientes ambulatorios con FC. Se encontró un efecto benéfico de ambas intervenciones, evidenciado en el aumento del delta del puntaje promedio de conocimiento alcanzado post-intervención y que este efecto se mantiene aunque disminuido hasta dos meses después de haber iniciado las intervenciones.

Sin duda alguna, la FC continúa siendo un problema de salud pública que debe ser abordado por los profesionales de la salud, implicados en su manejo. Estudios previos han establecido que los factores asociados a un bajo nivel de conocimiento y una pobre adherencia al tratamiento son: desconocimiento del proceso de la enfermedad, incumplimiento del régimen terapéutico, falta de apoyo social y seguimiento inadecuado ${ }^{(4)}$. Esto demuestra que la educación es un componente clave en el 
manejo de la enfermedad, y los profesionales de enfermería tienen un rol importante en la educación de los pacientes. En este sentido, cabe indicar que este estudio pudo demostrar que la educación de enfermería en este tipo de pacientes, independientemente de la estrategia (personalizada o telefónica) es útil y por lo tanto los profesionales de enfermería deberían ser incluidos en los equipos multidisciplinarios de atención.

De otro lado, en este estudio, la mediana de edad fue de 66 años con una edad mínima de 18 años y máxima de 87 años, lo cual indica al igual que otros estudios ${ }^{(11)}$, que la FC es una enfermedad del 'adulto mayor'. En el presente estudio se encontró que los pacientes mayores de 60 años tuvieron al final menor conocimiento acerca de su enfermedad. Dentro de este contexto, es válido indicar que la revolución de la longevidad en el siglo XXI ha dado paso a un aumento de la calidad y esperanza de vida, lo que otorga a los adultos mayores, una importancia especial para la sociedad. Sin embargo, en la "edad de la revolución de la longevidad", paradójicamente, los ancianos son una fuerza social desvalorada. Esto se convierte en una oportunidad, para que los futuros estudios y proyectos educativos en pacientes con FC sean diseñados y orientados para este tipo de población.

Por último, se recomienda seguir investigando en este tipo de población y se sugiere para estudios posteriores, evaluar el efecto de intervenciones educativas para la disminución de reingresos hospitalarios y mortalidad asociada a la descompensación de la FC, aumento en la calidad de vida y adherencia al tratamiento farmacológico y no farmacológico, teniendo en cuenta periodos de seguimiento prolongados. Desde el punto de vista de la profesión de enfermería, que cuenta con un lenguaje estandarizado: diagnósticos, intervenciones y resultados, se recomienda que los enfermeros y enfermeras continúen evaluando: la eficacia de las intervenciones, la validez clínica de los diagnósticos y la validez y confiabilidad de los resultados propios de la disciplina enfermera.

\section{Agradecimientos}

Este estudio fue financiado por la Vicerrectoría de Investigación y Extensión de la Universidad Industrial de Santander (código 56-71). Los autores también agradecen al Grupo de Investigación Observatorio Epidemiológico de Enfermedades Cardiovasculares de la Universidad Industrial de Santander, a los participantes del estudio y al personal directivo, administrativo y asistencial del Hospital Universitario de Santander.

\section{REFERENCIAS BIBLIOGRÁFICAS}

1. Cowie MR, Mosterd A, Wood DA, Deckers JW, Poole-Wilson PA, Sutton GC, et al. The epidemiology of heart failure. Eur Heart J 1997; 18(2):208-225.

2. Kosowsky J, Abraham WT, Storrow A. Evaluation and management of acutely decompensated chronic heart failure in the emergency department. Congest Heart Fail 2001; 7(3):124-136.

3.Jaarsma T, Stromberg A. Heart failure clinics in Europe. Prog Cardiovasc Nurs 2000; 15(2):67-68.

4.Ni H, Nauman D, Burgess D, Wise K, Crispell K, Hershberger RE. Factors influencing knowlewdge of and adherance to selfcare among patients with heart failure. Arch Intern Med 1999;159(14):1613-9. 
5. Strömberg A. The crucial role of patient education in heart failure. Eur $\mathrm{J}$ Heart Fail 2005;7:363-9.

6. Strömberg A. Educating nurses and patients to manage heart failure. Eur $\mathrm{J}$ Heart Fail 2002;1:33-40.

7. Lainscak M, Keber I. Validation of self assessment patient knowledge questionnaire for heart failure patients. Eur J Cardiovasc Nurs 2005 Dec;4(4):269-272. Epub 2005 Jun 3.

8. NANDA International. Diagnósticos enfermeros: definiciones y clasificación 20052006. Madrid: Harcourt; 2005.

9. McCloskey LC, Bulechek GM, editors. Clasificación de intervenciones de enfermería (CIEN), 3 ed. Madrid: Harcourt; 2000.

10. Johnson $M$, Maas $M$, Morread $S$, editors. Clasificación de resultados de enfermería (CRE), 3 ed. Madrid: Harcourt; 2005.

11. Yera-Casas AM, Mateos-Higuera del Olmo S, Ferrero-Loboy J, Páez-Gutiérrez TD. Evaluación de la intervención educativa al paciente anciano con insuficiencia cardíaca, realizada por enfermería a través de un plan de cuidados estandarizado. Enferm Clin 2009;19(4):191-8.

12. Phillips CO, Wright SM, Kern DE, Singa RM, Shepperd S, Rubin HR, et al. Comprehensive discharge planning with postdischarge support for older patients with congestive heart failure. A meta-analysis. JAMA. 2004;291:1358-67.

13. Boutron I, Moher D, Altman DG, Schulz K, Ravaud P, for the CONSORT group. Methods and Processes of the CONSORT Group: Example of an Extension for Trials Assessing Nonpharmacologic Treatments. Ann Intern Med. 2008:W60-W67.

14. Rojas-Sánchez OA, Rueda-Díaz LJ, Vesga-Gualdrón LM, Orozco-Vargas LC, Forero-Bulla CM, Camargo-Figuera FA. Eficacia de las intervenciones de enfermería para el diagnóstico "manejo inefectivo del régimen terapéutico". Enferm Clin 2009;19(6):299-305.

15. StataCorp. Stata statistical software: Release 11.0 College Station, TX: Stata Corporation; 2009.

16. Lauritsen JM, Bruus M, Myatt M. EpiData Entry Software. The EpiData Association, Odense-Denmark. 2008.

17. Fisher RA. The conditions under which $x 2$ measures the discrepancy between observation and hypothesis. J R Statist Soc 1924 May;87(3):442-50.

18. Vickers AJ, Altman DG. Analysing controlled trials with baseline and follow up measurements. BMJ 2001;323:1123-4.

19. World Medical Association Declaration of Helsinki. Ethical principles for medical research involving human subjects. Nurs Ethics 2002 Jan;9(1):105-9.

20. The Belmont Report. Disponible en:

http://www.hhs.gov/ohrp/humansubjects/guidance/belmont.html

Consultado: Septiembre 15, 2012.

21. Consejo de Organizaciones Internacionales de las Ciencias Médicas (CIOMS) / Organización Mundial de Salud. Pautas Éticas Internacionales para la Investigación Biomédica en Seres Humanos, Ginebra, 2002.

22. Normas de Buenas Prácticas Clínicas (BPC). Conferencia Internacional de Armonización (CIARM). Disponible en:

http://www.fda.gov/downloads/Drugs/GuidanceComplianceRegulatorylnformation/Guid ances/ucm073128.pdf

Consultado: Septiembre 15, 2012.

23. Ministerio de Salud. República de Colombia. Normas Científicas, Técnicas y Administrativas para la Investigación en Salud. Bogotá, Resolución 008430, Octubre 04,1993. 
24. Shapiro SS, Wilk MB. An analysis of variance test for normality (complete samples). Biometrika 1965;52(3-4):591-611.

25. Yehle KS, Sands LP, Rhynders PA, Newton GD. The effect of shared medical visits on knowledge and self-care in patients with heart failure: a pilot study. Heart Lung 2009 Jan-Feb;38(1):25-33. Epub 2008 Aug 30.

26.Strömberg A. Measuring patients' knowledge about heart failure. Eur J Cardiovasc Nurs 2005 Dec;4(4):267-8. Epub 2005 Nov 8.

27. Agård A, Hermerén G, Herlitz J. When is a patient with heart failure adequately informed? A study of patients' knowledge of and attitudes toward medical information. Heart Lung 2004 Jul-Aug;33(4):219-26.

28. van der Wal MH, Jaarsma T, Moser DK, Veeger NJ, van Gilst WH, van Veldhuisen DJ. Compliance in heart failure patients: the importance of knowledge and beliefs. Eur Heart J 2006 Feb;27(4):434-40. Epub 2005 Oct 17.

29. Falces C, López-Cabezas C, Andrea R, Arnau A, Ylla M, Sadurní J. Intervención educativa para mejorar el cumplimiento del tratamiento y prevenir reingresos en pacientes de edad avanzada con insuficiencia cardíaca. Med Clin (Barc). 2008;131(12):452-6. 